\title{
Unsteady Aerodynamic Analysis of a Bird-Damaged Turbofan
}

\author{
Eric R. Muir* and Peretz P. Friedmann ${ }^{\dagger}$ \\ Department of Aerospace Engineering, The University of Michigan, Ann Arbor, MI, 48109, USA
}

\begin{abstract}
The unsteady aerodynamic behavior of a bird-damaged fan stage at the inlet of a highbypass turbofan engine is examined with a computational fluid dynamics (CFD) aerodynamic model suitable for use in an aeroelastic response calculation. ANSYS CFX, a commercially available CFD solver, is utilized to perform the steady-state and unsteady CFD calculations for the undamaged and damaged fan. The damaged fan sector consists of 5 damaged blades that correspond to the geometry resulting from a bird strike. An automated mesh deformation scheme using radial basis function interpolation is developed to generate a high quality computational mesh for the damaged geometry. The steadystate calculations of the undamaged fan are verified against those from an industry CFD solver. The steady-state calculations of the damaged fan predict a flow loss of $14.7 \%$ with the operating point being near the stall point of the characteristic curve. Furthermore, significant separation and recirculation of the flow results from the damaged blades. The unsteady calculations of the damaged fan exhibit a periodic behavior in which the mass flow rate and total pressure ratio oscillate due to the progression and regression of a region of stalled flow emanating from the damaged sector.
\end{abstract}

\section{Nomenclature}

$\begin{array}{ll}B P R=\frac{\dot{m}_{L E_{B}}}{\dot{m}_{L E_{C}}} & \text { Bypass ratio } \\ C_{j} & \text { RBF interpolant coefficients } \\ D & \text { Number of RBF driver points } \\ \dot{m} & \text { Mass flow rate } \\ \dot{m}_{R} & \text { Referred mass flow rate } \\ N & \text { Number of fan blades } \\ P & \text { Total pressure } \\ P R=\frac{P_{B}}{P_{L E_{B}}} & \text { Total pressure ratio } \\ r & \text { Radial (spanwise) coordinate } \\ T & \text { Total temperature } \\ \mathbf{u} & \text { Deformation vector } \\ \hat{\mathbf{u}} & \text { RBF interpolant of the deformation } \\ x & \text { Axial coordinate, parallel with the engine centerline } \\ \mathbf{x}=(x, y, z) & \text { RBF evaluation point } \\ \mathbf{x}_{d}=(x, y, z) & \text { RBF driver point }\end{array}$

Greek Symbols

$\theta \quad$ Circumferential coordinate

$\phi \quad$ Radial basis function

*Ph. D. Candidate, Student Member AIAA.

${ }^{\dagger}$ Francois-Xavier Bagnound Professor of Aerospace Engineering, Fellow AIAA and AHS. 


$\begin{array}{ll}\text { Subscripts } & \\ B & \text { Evaluated at Station } B \\ L E & \text { Evaluated at Station } L E \\ L E_{B} & \text { Evaluated at Station } L E_{B} \\ L E_{C} & \text { Evaluated at Station } L E_{C}\end{array}$

Abreviations

CFD

FAA

FAR

FEM

RANS

$\mathrm{RBF}$

RBFN

$S T D$

\author{
Computational Fluid Dynamics \\ Federal Aviation Administration \\ Federal Aviation Regulation \\ Finite Element Method \\ Reynolds-averaged Navier-Stokes \\ Radial basis function \\ Radial basis function network \\ Standard atmosphere conditions
}

\section{Introduction}

Bird strike on jet engine fan blades is an important design consideration for both civilian and military aircraft. Bird strikes normally occur during takeoff and landing due to the prevalence of birds in proximity to the ground ${ }^{1}$ The low flight levels at which bird strikes occur limit recovery options and enhance the risk associated with bird strike events. The turbofan engines used in many commercial and military aircraft have a large intake area covered by fan blades that increases the chance of bird strikes. Furthermore, the thin, low aspect ratio, low camber fan blades used in turbofans are structurally and aerodynamically optimized for efficient operation at normal operating conditions, and bird damage induces off-design operation.22 Therefore, turbofan designs represent a sophisticated trade-off between structural integrity, propulsive efficiency, and robustness under bird strike.

During bird-strike, the bird collides with the engine fan, fragments, and propagates through the engine core and bypass duct. Such an impact can cause substantial deformation of the fan blade leading edge, global bending and twist of the blade, blade cracking and crack propagation, and fatigue failure. ${ }^{3}$ Furthermore, the structural mistuning and unsteady aerodynamic loading caused by bird damage induce fan blade vibrations driven by the coupled interaction of the unsteady, nonlinear aerodynamics and the structural dynamics, 4 producing a complex aeroelastic response problem, which can lead to aeroelastic instability $\sqrt[5]{6}$ Predicting the aeroelastic behavior of a bird damaged fan blade represents a significant design barrier in the development of improved-efficiency turbofan engines.

The Federal Aviation Administration (FAA) mandates comprehensive standards for bird strike resistance ${ }^{7}$ These standards require that each engine maintain $75 \%$ of the maximum rated thrust and meet engine handling requirements for a series of post bird strike operating conditions that simulate an emergency landing sequence. Engine certification is achieved through successful demonstration of compliance with Federal Aviation Regulations (FAR) Part 33 through complete-engine tests in which a bird is fired with an air cannon at a test stand mounted, running engine. Several engine tests are required to demonstrate compliance with regulations, and each test destroys a new engine. Therefore, the cost associated with engine certification for bird-strike is high.

While necessary for FAR compliance, complete-engine tests are infeasible within the design iteration process due to the associated cost. As a result, approximate component-based engine tests are performed to isolate the bird damage by firing a bird at a subset of the engine (typically the fan stage) that is rotated in a vacuum $13[8$ Such tests investigate the ability of the fan blades to retain their structural integrity during and after a bird strike event. A damaged fan stage is installed in an otherwise undamaged engine to assess the effect of bird-damaged fan blades on the engine aerodynamic performance and aeroelastic response of the fan blades. Experimental tests demonstrate that bird damage may reduce flutter margins and produce rotating stall, reinforcing the importance of an accurate bird-strike aeroelastic analysis. $[4$ 
Numerical simulations provide an affordable and cost effective alternative for assessing the aerodynamic loading and aeroelastic behavior of a deformed fan. However, the combined aerodynamic and structural modeling of a bird-damaged fan assembly, where the damage is typically isolated to a handful of blades, is a complex and challenging problem. The structural model must accommodate the mistuning of the birddamaged fan blades and the aerodynamic model must be time-accurate, nonlinear, viscous, and include a turbulence model to address time-dependent flow separation and reattachment ${ }^{9}$ The finite element method (FEM) is typically used to model the bird impact and resulting structural response since it can represent non-linear material behavior and complex geometries $[0] 16$ For the representation of the aerodynamic loading, computational fluid dynamics (CFD) is required to accurately capture the complex flow phenomena associated with bird damaged turbofans 17$] 18$ Reliable FEM and CFD methods exist to compute the bird impact, structural dynamic response, and unsteady aerodynamic loads of a damaged fan blade. However, due to the computational times required of FEM and CFD methods, the structural and aerodynamic computations are performed separately in an uncoupled or loosely coupled manner. Therefore, the aeroelastic effects that may be important in the bird strike problem may not be properly accounted for.

Despite its importance, few computational studies have examined the aerodynamic behavior and aeroelastic response of a bird-damaged fan. Bohari and Sayma ${ }^{19}$ present a CFD method to analyze the aerodynamic characteristics of a bird-damaged NASA rotor 67 containing a single blade with "assumed" leading edge damage. An automated procedure is used to map the characteristic curve of the damaged fan, and the stall boundary is determined at three engine operating speeds. Overall, the steady-state CFD results conclude that the stall margins deteriorate for the damaged fan with stall occurring below the design operating line. However, the mass flow rate and total pressure ratio produced by the fan were unaffected by the damaged blade.

In a unique set of studies, Imregun et al $\underline{49}$ examined the aeroelastic stability of a bird-damaged fan using a fully coupled CFD/FEM formulation in the time domain in which the form of the bird-damage is "assumed" and isolated to two blades. In Ref. 9, the steady-state CFD analysis indicates a change in the shock structure of the damaged blades as well as full flow separation and a strong recirculation area indicative of a wake being released from the damaged blades. In addition, considerable blockage of the flow field is apparent, and the mass flow rate dropped $8 \%$ compared to the undamaged fan assembly. The fully coupled analysis demonstrates instability of the first torsion mode of a damaged blade; however, it is unclear if the growth in modal displacement is the result of a flutter mechanism or the strong wake shed by the upstream damaged blade. In Ref. 4, the aeroelastic stability of the damaged assembly modes at $70 \%$ engine speed was computed and compared to the undamaged configuration. Overall, the aeroelastic stability of a bird-damaged assembly was found to be significantly poorer than the undamaged one, with significantly altered mode shapes due to gross structural mistuning. Also, it was found that a substantially damaged fan operating at high pressure ratios produces an unsteady flow response in which no steady-state solution can be achieved. Finally, aeroelastic stability was found to be sensitive to flight conditions with flutter stability being reduced at low pressure ratios and rotating stall occurring at high pressure ratios.

These studies provide insight into the aerodynamic behavior and aeroelastic response of a bird-damaged fan. However, the damage considered is not representative of the worse-case blade damage produced during bird-strike certification tests, which typically includes leading edge damage over a large region of the blade span accompanied by global bending and twist of the blade. The turbofan geometries in these studies did not resemble modern, high-bypass turbofans, and the damaged sector is limited to one or two blades. Furthermore, the aeroelastic response calculation of Imregun et al. was performed at $70 \%$ engine rotational speed where as bird-strike typically occurs during take-off at 100\% engine rotation speed.

For improved understanding of the bird-strike problem, a computational aeroelastic study of a bird damaged commercial turbofan operating at take-off conditions is needed, where the bird-damage is representative of experimental bird strike tests or accurate numerical simulation of the bird-strike event. A key component of such an aeroelastic analysis is a reliable aerodynamic model capable of representing the complex flow phenomena associated with bird-damaged turbofans. The purpose of this study is to describe an aerodynamic model suitable for use in an aeroelastic study of a bird-damaged commercial turbofan and present the steady-state and unsteady flow field resulting from the bird-damaged fan. The specific objectives of this study are:

1. Describe an aerodynamic model suitable for calculating the nonlinear, unsteady aerodynamic behavior of a bird damaged fan. 
2. Present an automated mesh deformation scheme capable of producing a high quality CFD mesh of the damaged geometry.

3. Verify the aerodynamic solution for an undamaged fan with data provided by industry.

4. Present the steady and unsteady aerodynamic environment associated with a fan containing 5 birddamaged blades that are representative of a practical configuration.

\section{Aerodynamic Solver}

In this study, ANSYS CFX, a commercially available aerodynamic solver is used to solve the compressible unsteady Reynolds-averaged Navier Stokes (RANS) equations. The ANSYS CFX code uses a finite volume numerical approach that yields a near second-order accurate spatial discretization. A second-order accurate backward Euler time-integration scheme is used for the unsteady calculations. Furthermore, the k- $\epsilon$ turbulence model is implemented, scalable wall functions are used to resolve the near-wall boundary layer, and the fluid is assumed to be ideal and calorically perfect.

\section{A. Computational Domain}

The turbofan geometry examined in this study is representative of a commercial turbofan. A CFD calculation of the flow through the entire turbofan engine is computationally prohibitive. However, a CFD analysis of an isolated fan stage is adequate for the prediction of the performance characteristics and unsteady aerodynamic environment associated with a bird-damaged fan. The fan stage begins downstream of the engine inlet, extends into the bypass duct and core duct, and includes a set of fan blades, a rotating hub, a stationary shroud, and a stationary splitter, as shown in Fig. 1. The fan stage geometry is given in cylindrical coordinates $(r, \theta, x)$. The hub, shroud, and splitter geometries are defined by two-dimensional curves in the $r-x$ plane, and the three-dimension hub, shroud, and splitter surfaces are obtained by full revolution of these curves about the engine centerline. The surfaces connecting the edges of the hub, shroud, and splitter define the inflow, bypass duct outflow, and core duct outflow boundaries of the computational domain. The fan blade geometry is specified by a set of constant-span cross-sectional curves, denoted blade profiles, as shown in Fig. 1. The three-dimension blade surface is obtained using spline fits through each blade profile in the spanwise direction. Finally, a clearance between the blade tip and shroud is specified to accurately account for the influence of the tip gap on flow losses and shock structure.

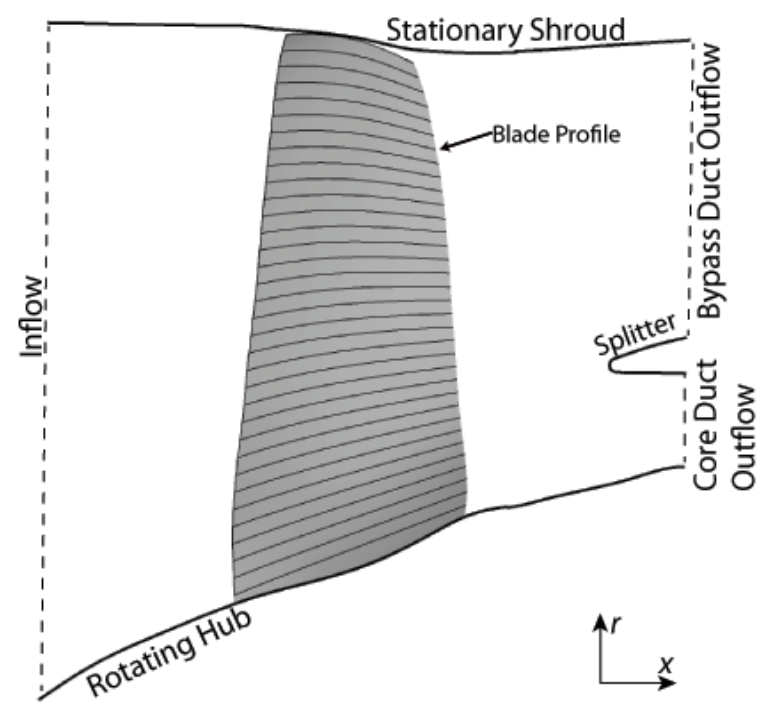

Figure 1: Meridional cross-section of the fan stage computational domain.

The computational domain consists of three sub-domains: the inlet, the passage, and the outlet, as depicted in Fig. 2(a) for a single blade passage of an undamaged fan and Fig. 2(b) for the full wheel. The full wheel computational domain consists of $N$ single blade passages copied and rotated about the engine 
centerline. The inlet sub-domain includes the downstream portion of the engine inlet nacelle and the engine nose cone. The passage sub-domain begins just upstream of the fan blade leading edge and surrounds the fan blades. The outlet sub-domain begins just downstream of the blade, extends into the bypass duct and core duct, and includes the upstream portion of the splitter. For an undamaged fan, radial symmetry can be exploited to reduce the computational domain. However, bird-damage destroys this symmetry and the whole-wheel fan stage must be modeled.

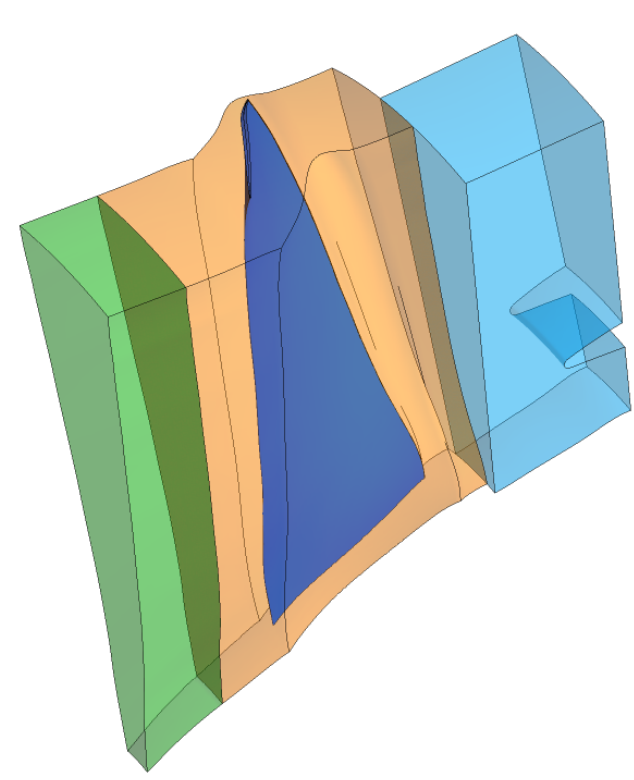

(a) Single blade passage computational domain.

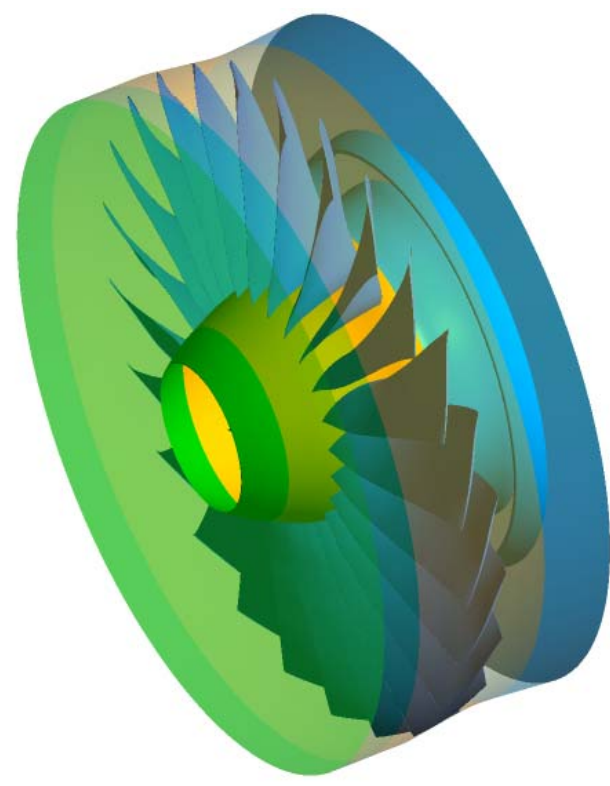

(b) Full wheel computational domain.

Figure 2: Fan stage computational domains: inlet (green), passage (orange), outlet (blue).

\section{B. Boundary Conditions}

Boundary conditions prescribe the aerodynamic properties of the flow on the external surfaces of the computational domain. At the domain inflow, the total pressure, total temperature, turbulence intensity, and flow direction of the incoming flow are enforced. The inflow total pressure, total temperature, and flow direction correspond to a particular flight condition and are specified as a function of radius from the engine centerline. The total pressure distribution includes flow losses due to the upstream engine inlet nacelle. At the core duct outflow, the mass flow rate is specified with the assumption that the engine core "pulls" a fixed mass flow rate through the fan for a given operating condition (free-stream condition and engine rotation speed). Static pressure is enforced at the bypass duct outflow using a radial-equilibrium condition that permits the static pressure to vary radially while maintaining the specified static pressure on average. Solid wall boundary conditions are enforced at the fan blades, hub, shroud, and splitter. At these boundaries, the velocity of the flow is set to match that of the wall through specification of a no-slip condition. The shroud and splitter constitute the stationary components of the domain in which a zero wall velocity is prescribed. The fan blades and hub constitute the rotating components of the domain in which a non-zero wall velocity equivalent to the engine rotation speed is prescribed.

\section{Operating Condition}

The performance of a fan stage is characterized by the total pressure ratio and referred mass flow rate produced by the fan. The total pressure ratio is defined as the ratio of the mass flow averaged total pressure at the bypass duct outflow to the mass flow averaged total pressure just upstream of the fan blades. The referred mass flow rate, calculated using Eq. (1), is the mass flow rate through the domain corrected for non-standard day inflow conditions and represents the mass flow that would pass through the fan if the inflow total pressure and total temperature corresponded to standard day conditions. The operating point 
of a fan stage is described by the total pressure ratio and referred mass flow rate resulting from operation at a particular operating condition defined by the engine rotation speed, inflow conditions, and outflow static pressure. The inflow total pressure and total temperature correspond to free-stream conditions, and the outflow static pressure represents the back-pressure produced by downstream engine components.

$$
\dot{m}_{R}=\dot{m} \sqrt{\frac{T}{T_{S T D}}}\left(\frac{P_{S T D}}{P}\right)
$$

A fan map depicts the operating points obtained using numerical simulations and experimental tests of an isolated fan stage for a variety of operating conditions. The operating points obtained with various outflow static pressures at a fixed engine speed and inflow conditions are connected to form characteristic curves. The stall point is identified by the peak in total pressure ratio along a characteristic curve and indicates the onset of stall. Stall is an undesirable, unsteady flow phenomena produced by flow separation that typically occurs at low mass flow rates and high bypass duct static pressure. A stall line connects the stall points on each characteristic curve and identifies the boundary of steady flow, where operating points to the left of the stall line are unsteady. A fan map also includes the fan operating line that consists of the unique set of operating points produced by the fan stage when installed in a complete engine. The operating line is measured during an infinitesimally slow throttle sweep of a complete-engine operating at a particular free-stream condition. Figure 3 provides a representative fan map that includes several characteristic curves, the associated stall points, and the stall line.

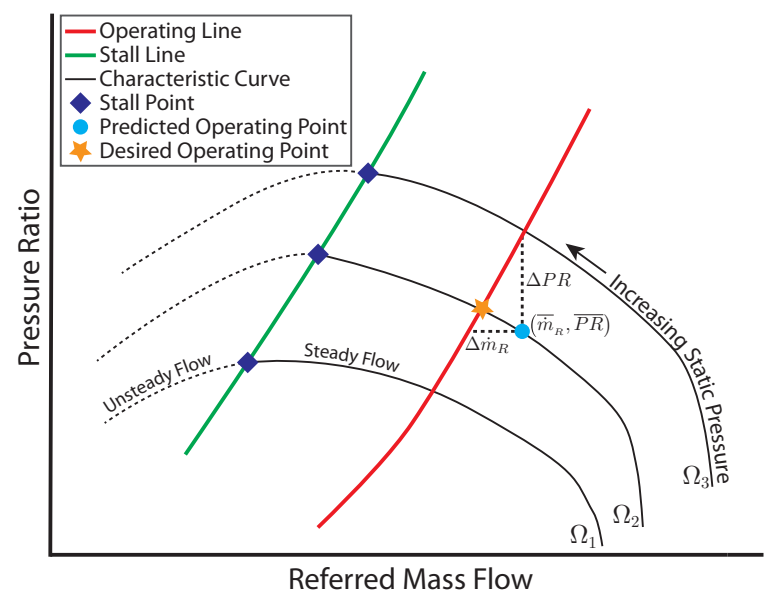

Figure 3: Representative Fan Map

For proper representation of the fan operating in a complete engine using a CFD model of an isolated fan stage, a bypass duct static pressure boundary condition is specified so that the predicted operating point coincides with a point on the operating line. The bypass duct static pressure necessary to achieve the desired operating point at the intersection of the characteristic curve and the operating line is unknown a priori. Therefore, an iterative procedure is utilized to map the characteristic curve and determine the bypass duct static pressure that yields an operating point within $1 \%$ error of the operating line. The error is calculated using Eq. (2) where the predicted operating point is denoted by $\left(\overline{\dot{m}}_{R}, \overline{P R}\right)$, and $\Delta \dot{m}_{R}$ and $\Delta P R$ denote the horizontal and vertical distance of the operating point from the operating line, as shown in Fig. 3.

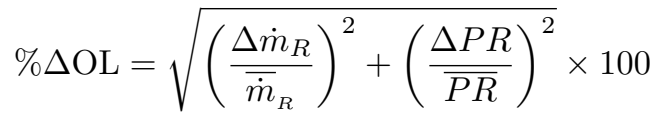

\section{Computational Mesh}

ANSYS TurboGrid is utilized to generate a high-quality, structured CFD mesh of hexahedral elements for the inlet and passage sub-domains of an undamaged single blade passage. A set of constant-span CFD mesh cross-sections are created based on a specified mesh topology and the three-dimensional mesh is constructed 
using spline fits and interpolation between each cross-section. ANSYS TurboGrid cannot accommodate geometries with a splitter that bisects the domain outflow; instead, ANSYS Meshing is used to create an unstructured hexahedral mesh for the outlet sub-domain. The CFD mesh of a single blade passage is copied and rotated to construct the full wheel CFD mesh of an undamaged fan.

Conventional mesh topologies, including H-Grid, J-Grid, C-Grid, L-grid, and O-grid, typically require a substantial amount of user manipulation to construct a CFD mesh of acceptable quality, particularly for complex blade geometries. Furthermore, these conventional topologies often result in an excessive mesh resolution within the blade passage in order to ensure a sufficient boundary layer resolution. ANSYS TurboGrid features an Automatic Topology and Meshing (ATM) optimized topology method that can be used to create high-quality, structured meshes without the constraints of conventional topologies. For this reason, the ATM topology is used in this study to generate a structured, hexahedral mesh of the inlet and passage domains of an undamaged single blade passage. The ANSYS TurboGrid ATM topology is not applicable for full wheel geometries that include a set of damaged blades. Instead, an automated mesh deformation scheme was developed to generate a high quality, full wheel CFD mesh for a fan stage that includes a set of damaged blades.

\section{A. Computational Mesh for the Undamaged Geometry}

The selection of mesh topology, mesh density, and boundary layer resolution for the undamaged CFD mesh are motivated by the physical flow phenomena expected and the degree of accuracy desired from the CFD solution. Proper resolution of the boundary layer and tip gap is essential to accurately capture the shock structure and flow losses due to viscosity in the boundary layer and flow leakage through the tip gap. Additionally, the mesh topology must avoid substantially skewed elements, areas of large expansion in element size, or elements with large aspect ratio to ensure numerical accuracy and solution convergence.

Three mesh resolutions are examined in this study: "coarse", "medium", and "fine". Figure 4(a) depicts a constant-span cross-section of the coarse mesh at $75 \%$ blade span for a single blade passage, and Fig. 4(b) depicts an overall meridional view of the computational mesh for the coarse mesh. The overall topology of the coarse, medium, and fine mesh are the same, with the primary difference being the boundary layer refinement and tip gap resolution. Figure 5 compares the leading-edge boundary layer refinement at $75 \%$ blade span for the coarse, medium, and fine meshes. The fine mesh is constructed with an average $y+$ value of $\approx 3$ for direct wall integration of the boundary layer. The coarse and medium mesh feature a larger $y+$ value and utilize scalable wall functions to reduce the computational expense associated with resolving the boundary layer flow. Table 2 lists the number of nodes in the CFD mesh, the number of nodes in the radial direction between the blade tip and the shroud, and the minimum, maximum, and area-average $y+$ value of the first node from the blade surface.

Table 2: Details of the coarse, medium, and fine meshes.

\begin{tabular}{l||c|c|c|c|c} 
Mesh & \# of Nodes & \# Tip Nodes & Min. $y+$ & Max. $y+$ & Avg. $y+$ \\
\hline Coarse & 435,546 & 4 & 1.76 & 400.86 & 101.6 \\
Medium & 625,485 & 9 & 0.91 & 129.0 & 24.6 \\
Fine & $1,278,743$ & 43 & 0.08 & 15.2 & 2.6
\end{tabular}

\section{B. Computational Mesh for the Damaged Geometry}

The damaged fan geometry considered in this study consists of 5 bird-damaged fan blades located in an otherwise undamaged fan. The blade damage resembles that resulting from an experimental bird strike test of a commercial turbofan in which a single 2.5 pound bird was ingested at take-off conditions. The damaged blade surface is described using a set of damaged blade profiles. Figures 6(a) and 6(b) depict the undamaged and damaged fan geometry for a 5-blade sector of the fan stage. Figure $6(\mathrm{~d})]$ depicts the undamaged and damaged blade profiles at $75 \%$ span corresponding to the overlaid undamaged and damaged fan geometry in Fig. 6(c). The blade damage includes substantial leading edge deformation of all 5 blades as well as global bending and twist. Furthermore, the damage covers over $50 \%$ of the blade span, with the largest deformation occurring for Blade 4. 


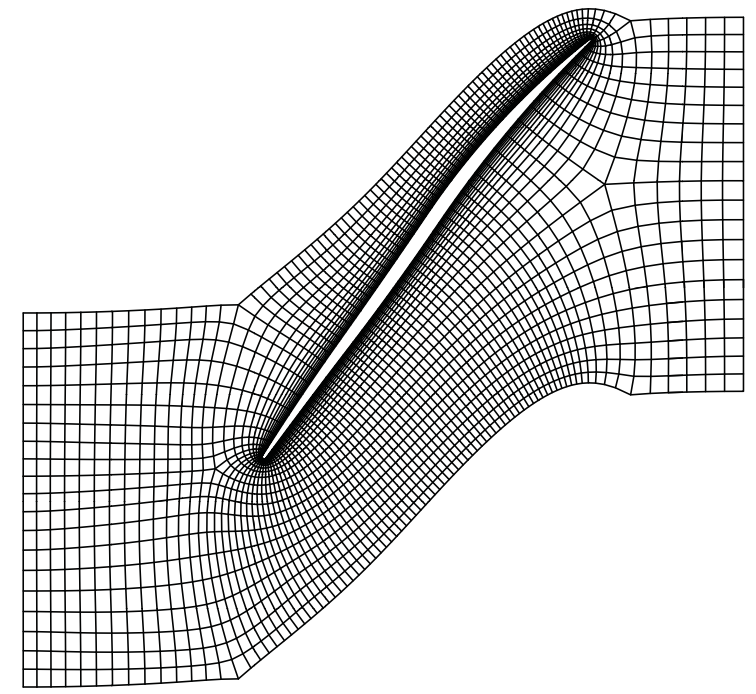

(a) Coarse mesh at $75 \%$ blade span.

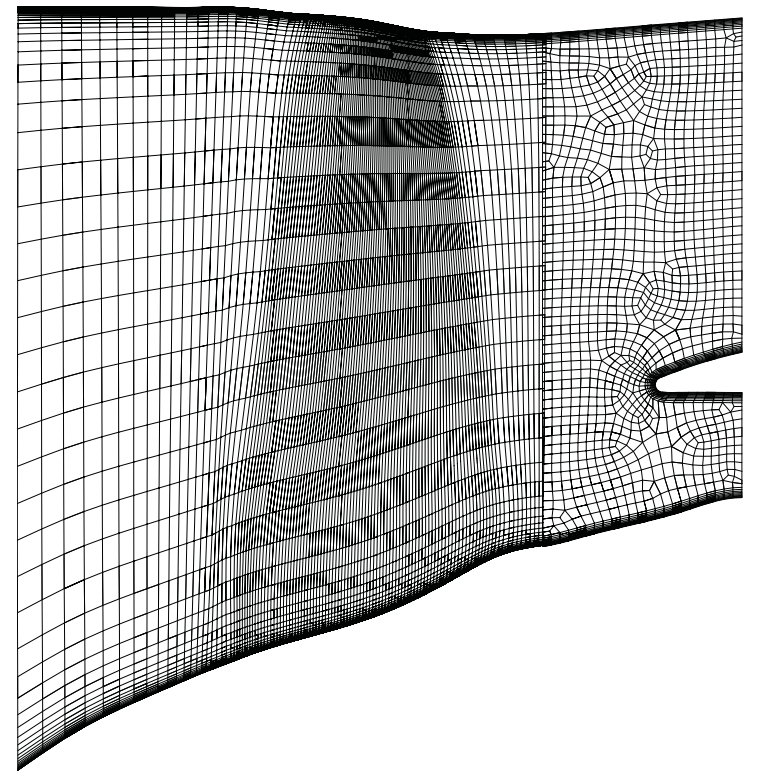

(b) Meridional view of coarse mesh.

Figure 4: Constant-span and meridional cross-sections of the coarse mesh.

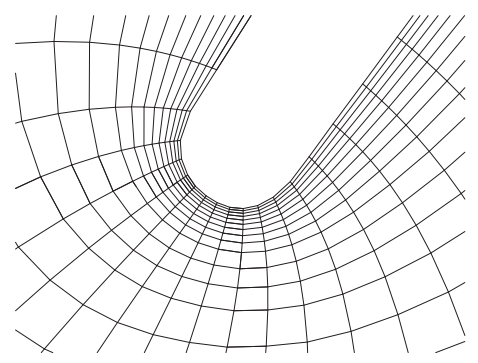

(a) Coarse mesh leading edge at $75 \%$ blade span.

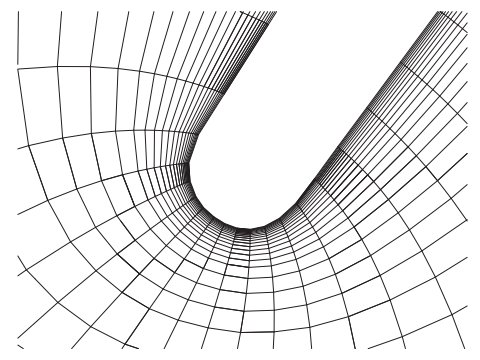

(b) Medium mesh leading edge at $75 \%$ blade span.

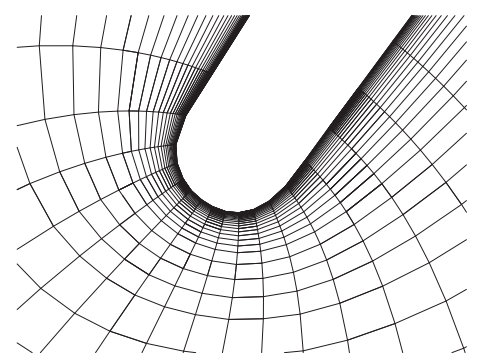

(c) Fine mesh leading edge at $75 \%$ blade span.

Figure 5: Comparison of leading-edge boundary layer resolution for the coarse, medium, and fine mesh at $75 \%$ span.

To extend the high mesh quality produced by the ANSYS Turbo Grid ATM mesh topology to the damaged fan geometry, an automated mesh deformation procedure was developed. This procedure employs a radial basis function network (RBFN) to interpolate the deformation from the bird damaged blades to the CFD mesh of the undamaged wheel. RBFN interpolation is an effective tool for multivariate interpolation and has been successfully utilized for large-amplitude mesh deformation in aeroelastic applications and CFD-based aerodynamic shape optimization studies. 20,25

A RBFN consists of a linear combination of radial basis functions (RBF) that are used to map the deformation prescribed at the fluid domain boundaries to the interior CFD mesh. A RBF, $\phi$, is a scalar function whose value depends only on the distance from the evaluation point, $\mathbf{x}$, to the origin, such that $\phi=\phi(\|\mathbf{x}\|)$. Provided a set of $D$ driver points at which the deformation is known, the RBF interpolant of the deformation field is constructed in the form given by Eq. (3). The RBF fitting coefficients, $C_{j}$ are uniquely determined by ensuring the deformation evaluated with the RBF interpolant at the driver points is equal to the prescribed deformation, as given in Eq. (4). The choice of RBF is important to ensure the best possible representation of the deformation field and resulting mesh quality. Reference 25 demonstrated that the volume spline defined by $\phi(\|\mathbf{x}\|)=\|\mathbf{x}\|$ is an ideal RBF for CFD mesh deformation; therefore, the volume 


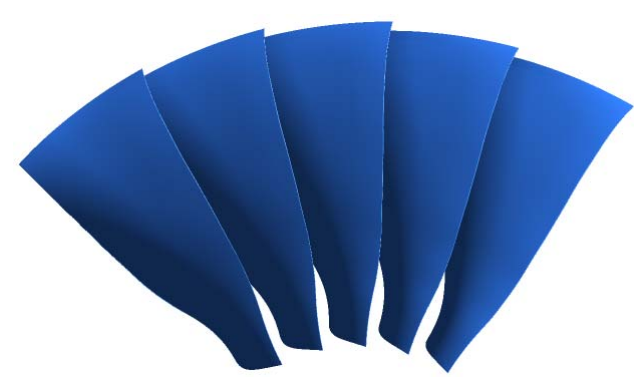

(a) Undamaged fan sector.

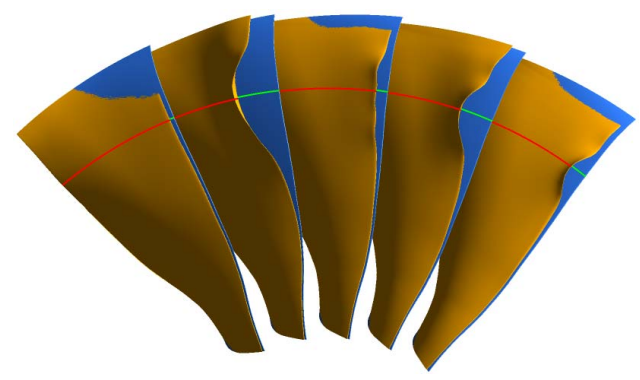

(c) Overlaid undamaged and damaged fan sector with blade profiles at $75 \%$ span.

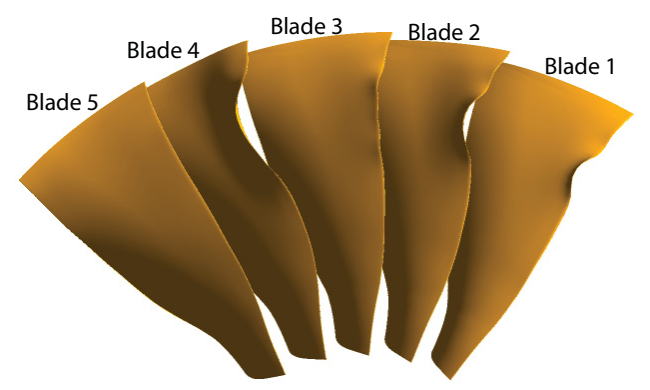

(b) Damaged fan sector.

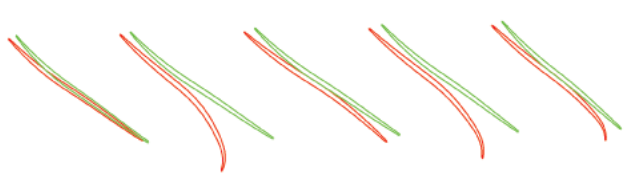

(d) Undamaged (green) and damaged (red) blade profiles at $75 \%$ span.

Figure 6: Undamaged and damaged fan geometry with blade profiles.

spline is used in this study where the norm is evaluated as the Euclidean distance: $\|\mathbf{x}\|=\sqrt{x^{2}+y^{2}+z^{2}}$.

$$
\begin{gathered}
\hat{\mathbf{u}}(\mathbf{x})=\sum_{j=1}^{D} C_{j} \phi\left(\left\|\mathbf{x}-\mathbf{x}_{d j}\right\|\right) \\
\mathbf{u}_{i}=\hat{\mathbf{u}}\left(\mathbf{x}_{d i}\right)=\sum_{j=1}^{D} C_{j} \phi\left(\left\|\mathbf{x}_{d i}-\mathbf{x}_{d j}\right\|\right) \quad 1 \leq i \leq D
\end{gathered}
$$

To generate the CFD mesh of the damaged geometry, Eq. (3) is used to map the deformation calculated between the undamaged and damaged blade profiles onto the undamaged CFD mesh. The points that define the undamaged blade profiles are chosen as the driver points, and the deformation vector at each driver point determined by calculating the difference between the points on the undamaged blade profiles and the corresponding points on the damaged profiles. The deformation is constrained at the inflow, outflow, hub, and shroud to preserve the profiles of these surfaces in the $(r, x)$ plane. Figure 7 depicts a crosssection of the undamaged and damaged coarse CFD mesh at $75 \%$ span. A key benefit of the automated mesh deformation procedure developed for this study is that a CFD mesh can be generated for a variety of damaged configurations using the same undamaged CFD mesh.

Several metrics are examined to quantitatively assess the high mesh quality preserved by the mesh deformation scheme: the minimum and maximum face angle, the element volume ratio, and the minimum element volume. The face angle is a measure of mesh skewness and is calculated as the angle between two element faces that share a common node. The element volume ratio is a measure of the local element expansion and is defined as the ratio of the maximum volume of an element that touches a node to the minimum volume of an element touching the same node. Finally, the minimum volume is the smallest element volume in the mesh and must be greater than 0 . Table 3 provides the limit for each mesh metric, as recommended by the ANSYS CFX literature, the extremum value of that metric within the mesh, as well as the percentage of elements in the damaged mesh that violate the metric. Clearly, the damaged CFD mesh produced by the the RBF mesh deformation procedure exhibits a high mesh quality with only a small percentage of elements violating the recommended limits for maximum and minimum face angle in the damaged medium and fine meshes. 


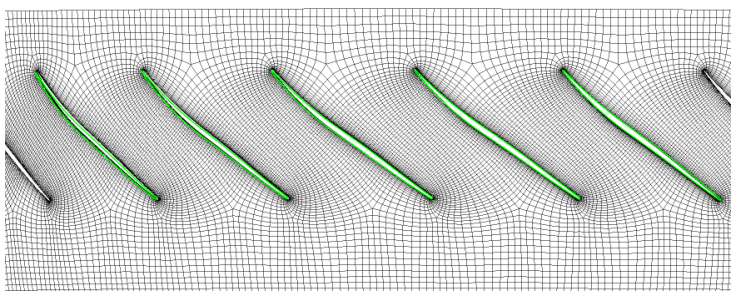

(a) Undamaged coarse mesh at $75 \%$ span with undamaged blade profiles (green).

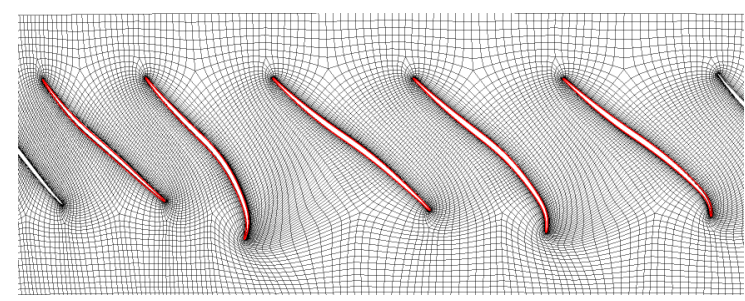

(b) Damaged coarse mesh at $75 \%$ span with damaged blade profiles (red).

Figure 7: Comparison of the undamaged and damaged coarse mesh at $75 \%$ span.

Table 3: Mesh quality metrics for the undamaged and damaged coarse, medium, and fine meshes.

\begin{tabular}{l|l||c|c||c|c||c|c}
\multicolumn{1}{l||}{} & \multicolumn{2}{c||}{ Damaged Coarse Mesh } & \multicolumn{2}{c||}{ Damaged Medium Mesh } & \multicolumn{3}{c}{ Damaged Fine Mesh } \\
Metric & Limit & Value & $\%$ Over & Value & $\%$ Over & Value & $\%$ Over \\
\hline Max. Face Angle & $>170^{\circ}$ & $140^{\circ}$ & $0.0 \%$ & $176^{\circ}$ & $0.1 \%$ & $180^{\circ}$ & $0.3 \%$ \\
Min. Face Angle & $<10^{\circ}$ & $44.1^{\circ}$ & $0.0 \%$ & $3.92^{\circ}$ & $0.1 \%$ & $0.52^{\circ}$ & $0.3 \%$ \\
El. Volume Ratio & $>30$ & 11.7 & $0.0 \%$ & 34.1 & $\approx 0.0 \%$ & 83.7 & $0.0 \%$ \\
Min. Volume & $<0 \mathrm{in}^{3}$ & $2.37 \mathrm{E}-8 \mathrm{in}^{3}$ & $0.0 \%$ & $8.26 \mathrm{E}-10 \mathrm{in}^{3}$ & $0.0 \%$ & $1.62 \mathrm{E}-11 \mathrm{in}^{3}$ & $0.0 \%$
\end{tabular}

\section{Results}

This section presents the aerodynamic results obtained with ANSYS CFX for the undamaged fan. In addition, the steady and unsteady aerodynamic behavior of a bird-damaged fan is examined to illustrate the capabilities of the aerodynamic model and provide insight into the aerodynamic behavior of a bird-damaged fan. For this study, the freestream conditions correspond to standard day $+27^{\circ} \mathrm{F}$ conditions, the engine rotational speed is set to take-off speed, and the flight Mach number is zero.

\section{A. Verification of Undamaged Fan Aerodynamic Calculations}

The aerodynamic calculations from ANSYS CFX for the undamaged fan stage are compared to results obtained with an industry CFD solver through comparison of overall flow parameters for the fan stage, radial flow distributions at various axial locations, and blade cross-section pressure distributions. The flow unsteadiness for an undamaged fan stage operating at design conditions is insignificant; therefore, only the steady-state aerodynamic results are compared. Furthermore, the ANSYS CFX solutions calculated with the coarse, medium, and fine meshes are nearly identical; therefore, only the results obtained with the coarse mesh are presented.

\section{Comparison of overall flow parameters}

Averaged values of several flow parameters are compared at various axial stations within the computational domain. The overall flow parameters, listed in Table 4 provide a picture of the overall flow field and are useful for comparison purposes. Station $L E$ refers to the axial location "just" upstream of the fan blade leading edge, and Station $B$ refers to the outflow of the computational domain in the bypass duct. Station $L E$ is composed of two sub-stations: Station $L E_{C}$ and Station $L E_{B}$. Station $L E_{C}$ is the portion of Station $L E$ through which flow travels into the core duct, and Station $L E_{B}$ is the remaining portion of Station $L E$ through which flow travels into the bypass duct. Table 4 also provides the L1 relative error norm of the flow parameters for comparison of the ANSYS CFX solution with the industry CFD solver results. The ANSYS CFX solution agrees very well with the industry CFD solver results, with the maximum error being less than $1 \%$. 
Table 4: Comparison of overall flow parameters.

\begin{tabular}{c|c} 
Parameter & $\begin{array}{c}\text { \% Error w/ } \\
\text { Industry Solver }\end{array}$ \\
\hline \hline$P_{L E}$ & $0.12 \%$ \\
$P_{L E_{C}}$ & $0.09 \%$ \\
$T_{L E_{C}}$ & $0.03 \%$ \\
$P_{L E_{B}}$ & $0.31 \%$ \\
$T_{L E_{B}}$ & $0.09 \%$ \\
$P_{B}$ & $0.60 \%$ \\
$T_{B}$ & $0.05 \%$ \\
$\dot{m}_{L E}$ & $0.21 \%$ \\
$\dot{m}_{L E_{B}}$ & $0.09 \%$ \\
$\dot{m}_{L E_{C}}$ & $0.76 \%$ \\
$\dot{m}_{L E}$ & $0.64 \%$ \\
$B P R$ & $0.63 \%$ \\
$P R$ & $0.90 \%$
\end{tabular}

\section{Comparison of radial flow distributions}

Radial distributions of several normalized flow quantities calculated with ANSYS CFX are compared to the industry CFD solver results. These radial distributions are calculated at the domain inflow (IN), $5 \%$ of the blade chord upstream of the blade leading edge (LE5), $5 \%$ of the blade chord downstream of the blade trailing edge (TE5), and the domain outflow (OUT). Figure 8 depicts the radial distributions of circumferentially-averaged axial velocity, static pressure, total pressure, and total temperature, normalized by their respective average values, at the four axial locations for the ANSYS CFX and the industry CFD solver results. The L1 relative error norm between the ANSYS CFX and industry CFD solver result is indicated by the labels on the plots.

Overall, the ANSYS CFX solution agrees very well with the solution from the industry CFD solver, with an average error of $0.70 \%$ for all radial distributions. The largest discrepancy occurs in the axial velocity and static pressure distribution at the OUT location in the core duct. This discrepancy may be due to differences in the implementation of the core duct mass flow boundary condition in the CFD solvers. This discrepancy is deemed acceptable, since the maximum error in the axial velocity and static pressure distributions at $O U T$ is only $3.27 \%$ and $1.12 \%$ respectively.

\section{Comparison of blade cross-sectional pressure distribution}

The final component in this verification effort is comparison of the chord-wise static pressure distributions at several blade cross-sections. Figure 9 depicts the chord-wise static pressure distribution, normalized by the average value, obtained with ANSYS CFX and the industry CFD solver at 30\%,60\%, and 90\% blade span. The L1 relative error norm between the two solutions is indicated by the labels on the plots. Good agreement in the chord-wise static pressure distributions between the ANSYS CFX and the industry CFD solver results is evident, with the minimum and maximum error being $0.93 \%$ and $3.14 \%$, respectively. There is also agreement in the chord-wise location of the passage shock, which influences the cross-section moment

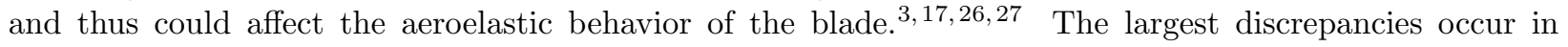
the leading edge and trailing edge pressure spikes and the pressure gradient at the passage shock. The main source of these discrepancies is attributed to the difference in mesh resolutions along the blade, which significantly affects the resolution of steep pressure gradients. Specifically, the static pressure jump across the passage shock in the industry CFD solver solution is somewhat smeared out due to the coarse chord-wise mesh resolution used in the industry CFD solver. The mesh used with ANSYS CFX has a finer chord-wise mesh resolution, and the static pressure jump across the passage shock is more sharply defined. 


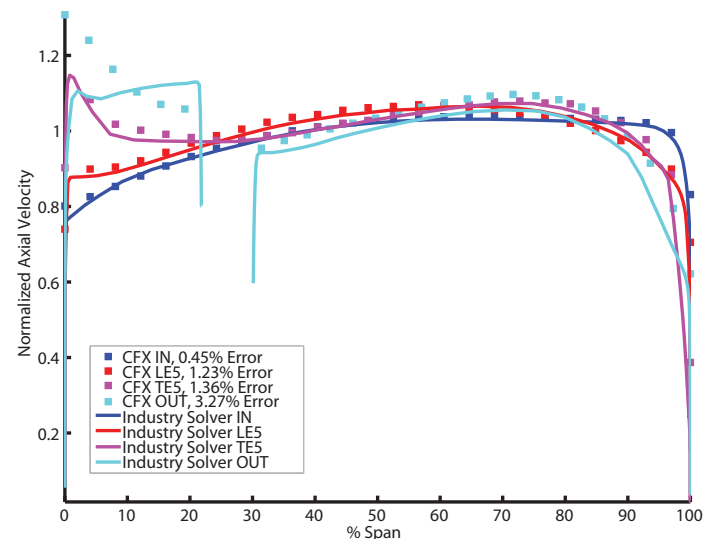

(a) Normalized axial velocity distributions at various axial locations.

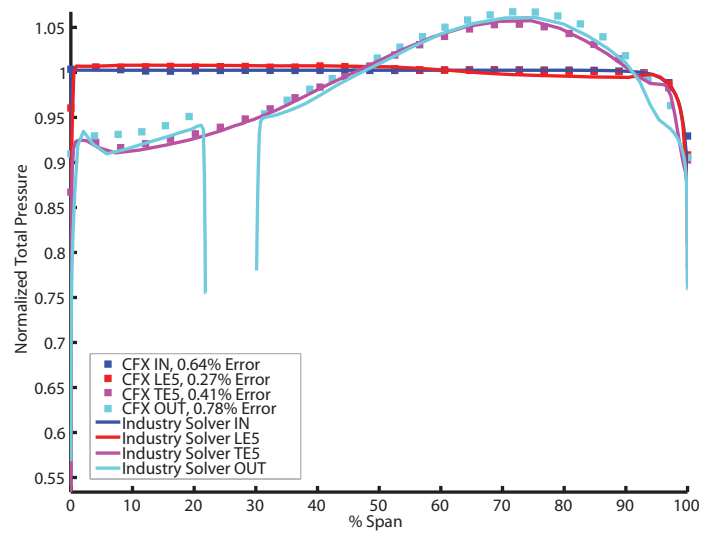

(c) Normalized total pressure distributions at various axial locations.

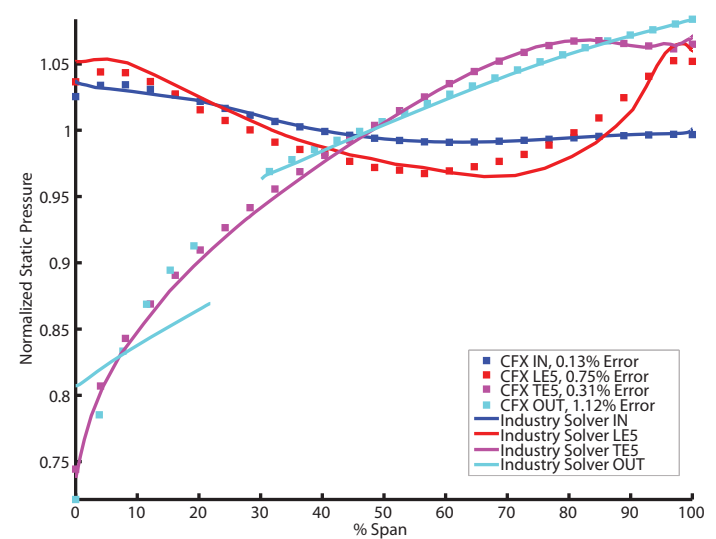

(b) Normalized static pressure distributions at various axial locations.

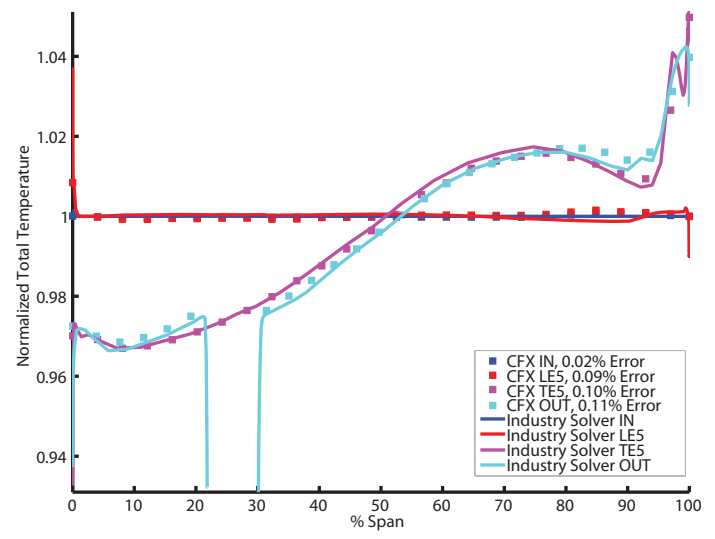

(d) Normalized total temperature distributions at various axial locations.

Figure 8: Comparison of radial distributions of several normalized flow quantities.

\section{B. Steady-State Aerodynamic Calculations of the Damaged Fan}

The steady-state aerodynamic results for the bird-damaged fan performed with the deformed coarse, medium, and fine meshes are presented in this section. The coarse mesh is used to map the characteristic curve of the damaged fan and identify the operating point that coincides with the operating line. The flow field associated with this operating point is compared with the steady-state analysis obtained using the industry CFD solver. Finally, a comparison of the flow field corresponding to the coarse, medium, and fine meshes is included to determine the importance of boundary layer and tip gap resolution in the steady-state aerodynamic analysis of a bird-damaged fan.

\section{Steady-state operating point of the damaged fan}

The characteristic curve of the damaged fan was mapped to achieve an operating point within $1 \%$ error of the fan operating line. The characteristic curve shows that the bird-damaged fan is operating very near the stall point, indicating that unsteady flow phenomena may be significant in the aerodynamic behavior of the bird damaged fan. A characteristic curve was not calculated using the medium or fine meshes due to the increased computational resources required to compute multiple operating points with these meshes. Instead, the bypass duct static pressure associated with the coarse mesh operating point was used to calculate the operating point with the medium and fine meshes. Figure 10 provides the fan operating line normalized by the undamaged operating point along with the damaged fan characteristic curve and the damaged fan operating points calculated with the coarse, medium, and fine mesh and the industry solver. The damaged 


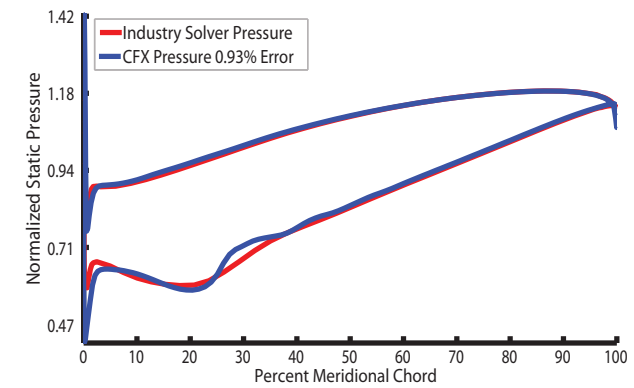

(a) Normalized chord-wise static pressure distributions at $30 \%$ blade span.

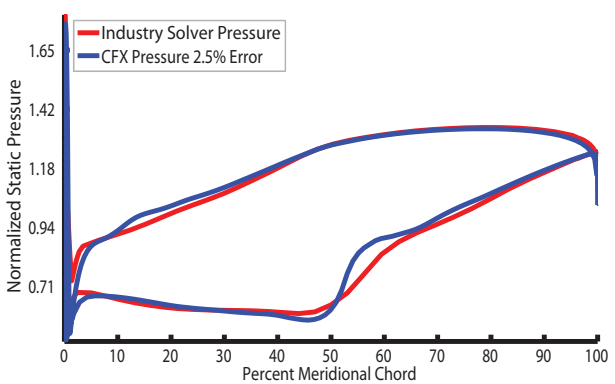

(b) Normalized chord-wise static pressure distributions at $60 \%$ blade span.

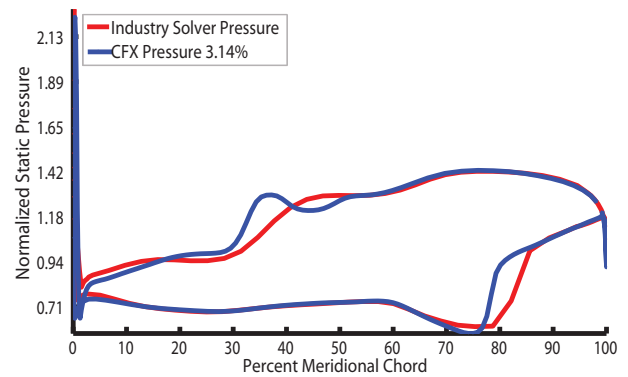

(c) Normalized chord-wise static pressure distributions at $90 \%$ blade span.

Figure 9: Comparison of normalized chord-wise static pressure distributions.

operating points calculated with the coarse, medium, and fine mesh all lie within $1 \%$ error of the operating line.

The operating point of the fan stage is significantly affected by the damaged blades. The total pressure ratio and referred mass flow both decrease by approximately $15 \%$ due to the bird damage, where the mass flow loss is directly related to the thrust loss resulting from the bird-strike. Table 5 lists the damaged operating points obtained with the coarse, medium, and fine meshes and the industry CFD solver normalized by the undamaged operating point along with the percent mass flow loss relative to the undamaged case. The slightly larger flow loss associated with the medium and fine meshes relative to the coarse mesh is attributed to the increased flow losses captured by the increased mesh resolution in the boundary layer and tip gap. In addition, the coarse, medium, and fine meshes predict a mass flow loss that is $1.9 \%-2.9 \%$ greater than the industry solver. Considering the inherent unsteadiness associated with the damaged fan operating near the stall point, the slight variations in operating point between the ANSYS CFX solver and the industry CFD solver are acceptable.

Table 5: Comparison of operating points for the undamaged and damaged fan.

\begin{tabular}{l||l||l|l|l|l} 
Solver & Configuration & $\begin{array}{l}\text { Normalized } \\
\dot{m}_{2 A_{R}}\end{array}$ & $\begin{array}{l}\text { Normalized } \\
P R\end{array}$ & $\begin{array}{l}\% \text { Flow } \\
\text { Loss }\end{array}$ & $\% \Delta \mathrm{OL}$ \\
\hline \hline Industry Solver & Damaged & 0.871 & 0.875 & $12.8 \%$ & $0.87 \%$ \\
ANSYS CFX & Damaged: Coarse & 0.851 & 0.853 & $14.7 \%$ & $0.45 \%$ \\
ANSYS CFX & Damaged: Medium & 0.842 & 0.852 & $15.7 \%$ & $0.65 \%$ \\
ANSYS CFX & Damaged: Fine & 0.845 & 0.850 & $15.4 \%$ & $0.09 \%$
\end{tabular}




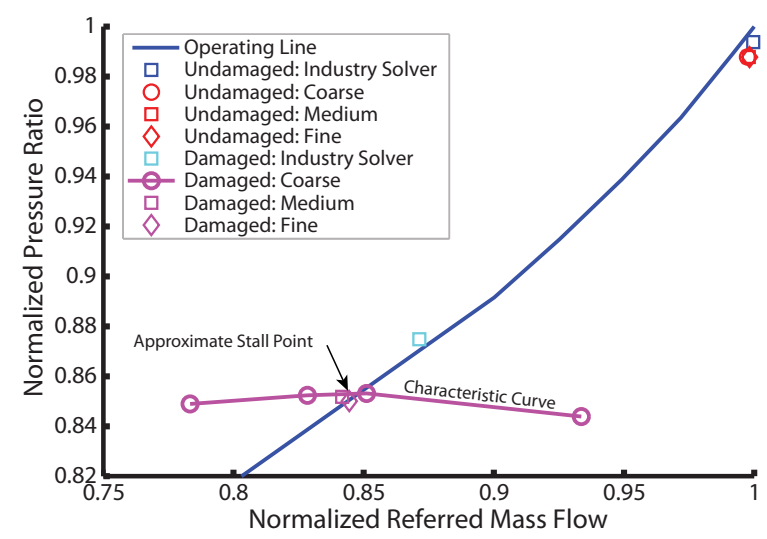

Figure 10: Normalized fan map including the operating line, undamaged operating points, damaged operating points, and damaged characteristic curve.

\section{Steady-state Mach number contours of the damaged fan}

To gain insight into the flow behavior resulting from the damaged fan sector, a constant-span circumferential slice of the wheel is examined and compared to the undamaged case. Figure 11] provides a Mach number contour of the damaged fan at $77 \%$ span, the location of greatest damage, obtained with the industry CFD solver and ANSYS CFX using the coarse, medium, and fine mesh. The five damaged blades are labeled as Blade 1 through Blade 5 where Blade 1 corresponds to the leading damaged blade. Overall, the ANSYS CFX solutions agree well with the industry solver in predicting the complex flow field associated with the damaged fan sector; however, a greater degree of separated flow and flow blockage is apparent in the ANSYS CFX solutions, which is likely responsible for the greater flow loss predicted by the ANSYS CFX solutions. Furthermore, the increased mesh resolutions of the medium and fine mesh have negligible effect on the solution at $77 \%$ span.

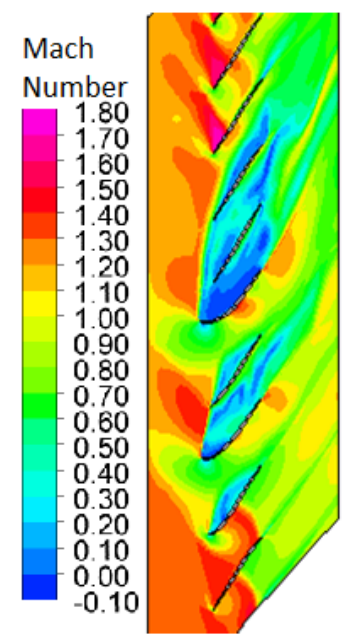

(a) Industry CFD solver.

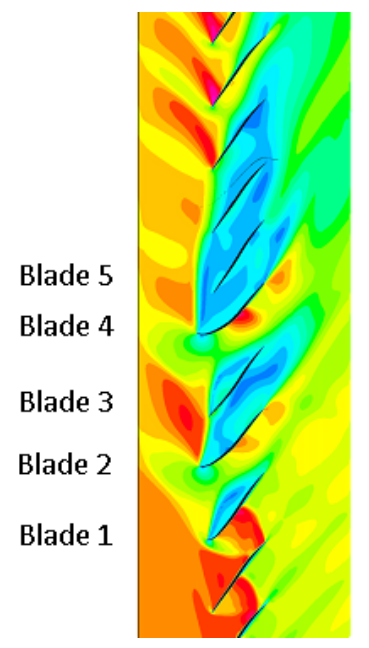

(b) ANSYS CFX: Coarse mesh.

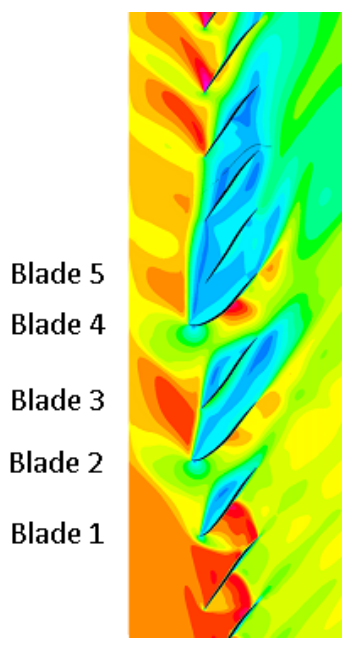

(c) ANSYS CFX: Medium mesh.

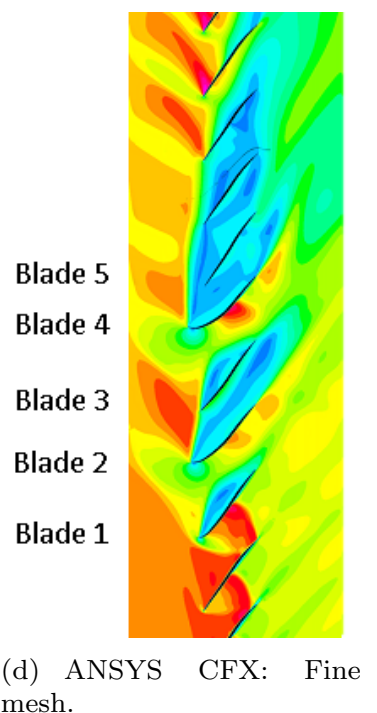

(d) ANSYS CFX: Fine mesh.

Figure 11: Comparison of steady-state Mach number contours of the damaged fan at $77 \%$ span.

When compared with the Mach number contour at 77\% span for the undamaged fan, depict in Fig. 12(b)] Fig. 11 clearly illustrates the significant effect of the bird damaged blades on the flow field of the entire fan. The moderate leading edge deformation of Blade 1 creates a separation bubble on the suction side of the blade that partially blocks the flow through the downstream passage. The larger leading edge deformation and global bending of Blade 2 and Blade 4 result in a more substantial flow disruption in which the flow 
through several downstream passages is blocked. Furthermore, the mass flow through the blade passages downstream of the damaged sector experience a loss of mass flow due to increased angles of attack and flow separations, and the passages upstream of the damaged sector compensate for this loss with an increased mass flow and a stronger passage shock.

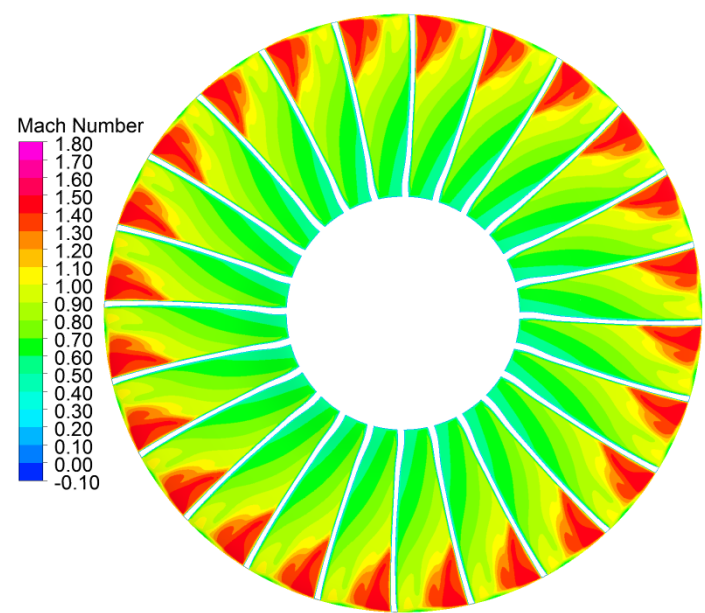

(a) Undamaged fan Mach number contour at mid-chord (direction of rotation: counter-clockwise).

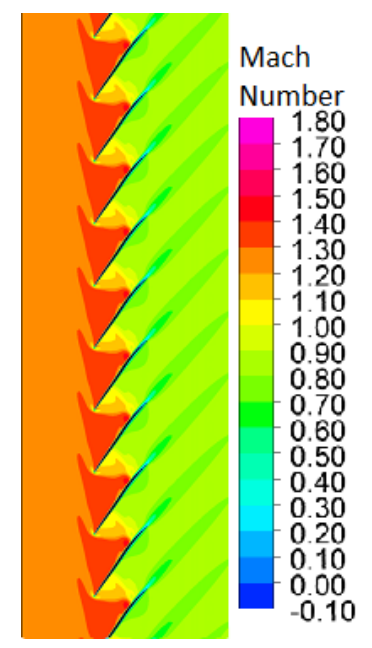

(b) Undamaged fan Mach number contour at $77 \%$ span.

Figure 12: Undamaged fan Mach number contours obtained with the coarse mesh.

Figures $12(\mathrm{a})$ and 13 provide Mach number contours on a constant-axial slice of the wheel at mid blade chord for the undamaged and damaged fans, respectively. Once again, the ANSYS CFX solutions obtained with the coarse, medium, and fine mesh show a negligible effect of the boundary layer and tip gap mesh resolution. Furthermore, when compared to the industry solver, the ANSYS CFX solutions predict the same overall flow features, especially in the blade passages adjacent to the damaged blades. However, the industry solver predicts a region of stalled flow at the blade tips that covers roughly $3 / 4$ of the fan wheel. In contrast, the ANSYS CFX solutions predict stalled flow for a larger spanwise portion of the blade tips but for only half of the fan wheel. As a result, the flow loss predicted by the industry solver is more evenly distributed across the fan, whereas the flow loss predicted by ANSYS CFX is concentrated to half of the fan wheel. In addition, the flow loss associated with the stalled blade tips is compensated by the increased flow through the upstream blade passages, as evident by the increased Mach number distribution through these blade passages. In both cases, the effect of the damaged sector is evident for the entire fan wheel, and the blade damage has a significant effect on the aerodynamic environment of the fan.

\section{Unsteady Aerodynamic Calculations of the Damaged Fan}

This sub-section presents the unsteady aerodynamic calculations of the bird-damaged fan using the RBF deformed coarse mesh. Unsteady calculations using the medium and fine meshes were not performed due to the increased computation times associated with the larger mesh sizes and very small effect of mesh density observed in the steady-state calculations. The steady-state solution is used to initialize the unsteady calculations, and the aerodynamic boundary conditions correspond to the steady-state values. A physical time-step of $\Delta t=\frac{1}{500 \Omega}$ is specified for 500 unsteady time-steps per revolution, and 3 sub-iterations are performed at each time-step to ensure convergence of the solution. The unsteady calculations were performed for 13,500 time-steps corresponding to 27 revolutions of the fan.

\section{Unsteady operating point of the damaged fan}

The total pressure ratio and referred mass flow rate, normalized by the respective undamaged value, are presented as a function of fan revolutions in Fig. 14(a) for the entire duration of the unsteady calculation. After the transients due to initialization of the unsteady calculation subside, the total pressure ratio and 


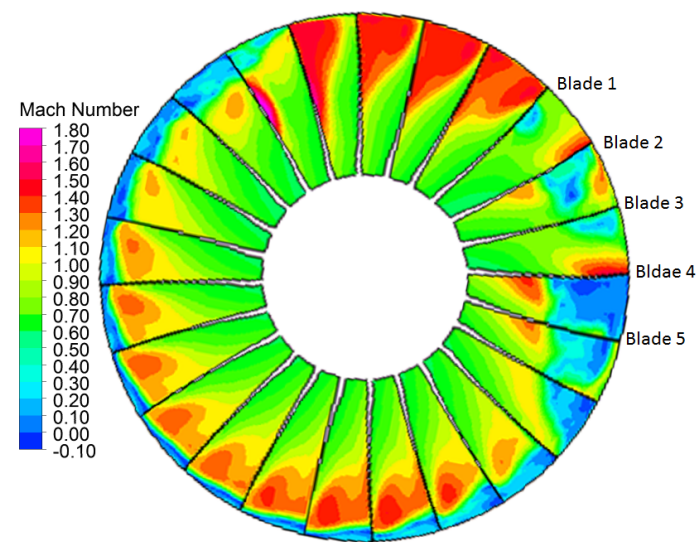

(a) Industry solver.

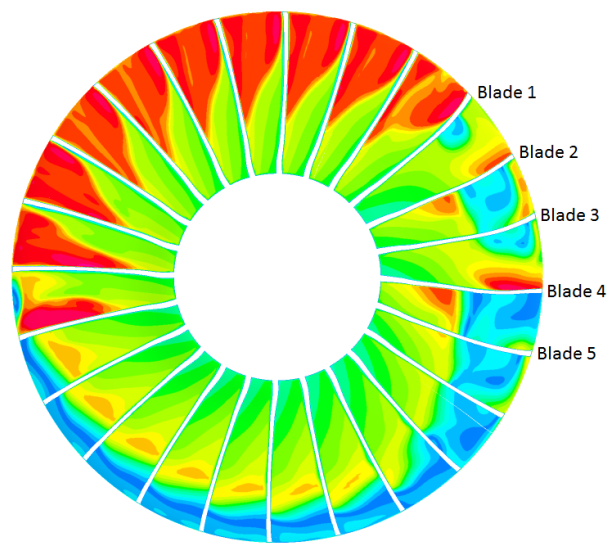

(c) ANSYS CFX: Medium mesh.

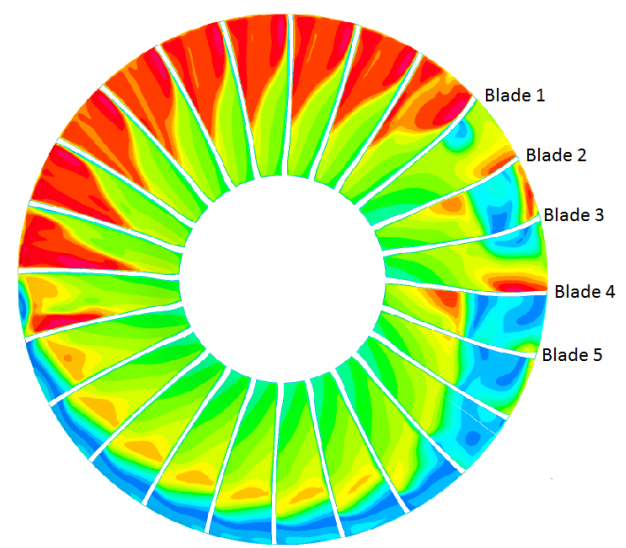

(b) ANSYS CFX: Coarse mesh.

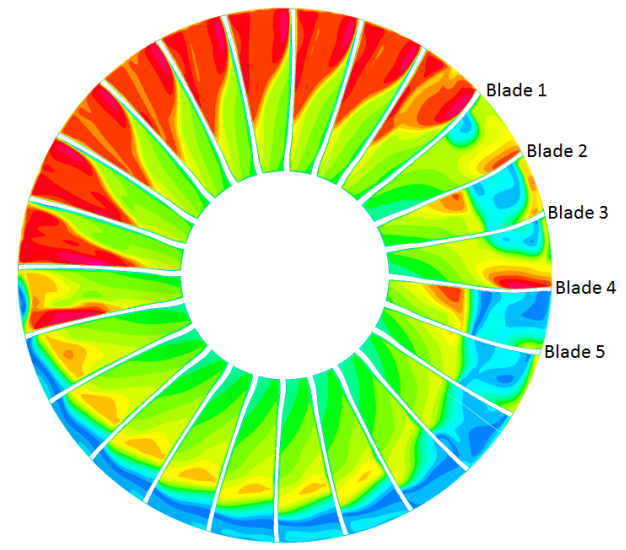

(d) ANSYS CFX: Fine mesh.

Figure 13: Comparison of steady-state Mach contours of the damaged fan at mid-chord (direction of rotation: counter-clockwise).

referred mass flow rate oscillate with a period of 6.2 revolutions. Overall, considerable unsteadiness in the operating point is apparent, with the referred mass flow oscillating $\pm 3.6 \%$ about its mean value and the total pressure ratio oscillating $\pm 1.3 \%$ about its mean value. Furthermore, the mean flow loss is $17.0 \%$, which is slightly greater than predicted by the steady-state calculation, and varies from $14.0 \%$ to $20.0 \%$ over the course of one oscillation. Figure 14(b) depicts the unsteady operating point plotted on the fan map along with the steady-state operating points and characteristic curve. The unsteady operating point oscillates about a point on the characteristic curve above the operating line, indicating that the unsteady effects contribute additional flow losses not captured by the steady-state calculation.

\section{Unsteady Mach number contours of the damaged fan}

Figure 15 depicts the unsteady Mach number contours at $77 \%$ span for 8 equally spaced time-steps spanning one representative oscillation of the unsteady solution. The corresponding total pressure ratio and referred mass flow rate at these time-steps are indicated by the fine vertical lines in Fig. 14(a). Little unsteadiness is observed in the near vicinity of the damaged blades where the flow remains largely separated with the flow through the damaged blade passages partially or totally blocked. In the blade passages directly downstream of the damaged sector, small oscillations in the flow separation point and degree of separation are evident, indicative of an unsteady wake being shed from the stalled blades.

The greatest level of flow unsteadiness occurs in the blade passages between Blade 15 and Blade 20. At 21.8 revolutions, the stalled flow emanating from the damaged sector, denoted a stall cell, covers roughly half of the fan wheel, from Blade 1 to Blade 15, and the mass flow rate is at a maximum. From 22.7 revolutions 


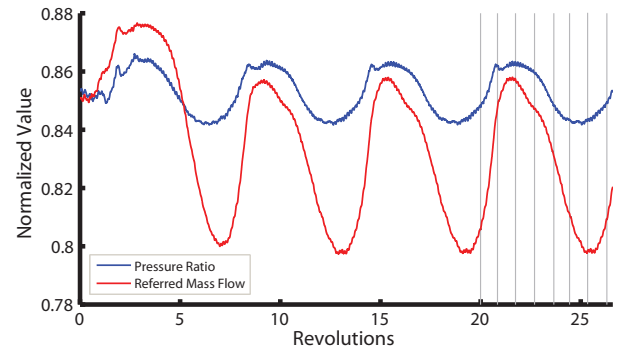

(a) Referred mass flow rate and total pressure time history.

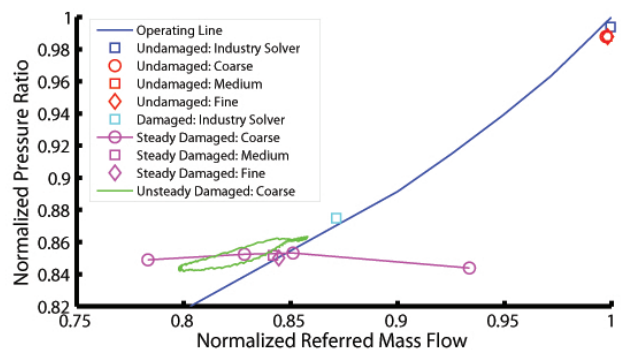

(b) Fan map with unsteady operating point.

Figure 14: Unsteady total pressure ratio and referred mass flow rate.

to 26.3 revolutions, the stall cell propagates opposite the direction of rotating until $3 / 4$ of the blade tips are stalled, from Blade 1 to Blade 19. At this point, the mass flow rate is at a minimum due to the partially blocked blade passages associated with the stalled flow. Subsequently, the stall cell rapidly regresses in the direction of rotation and the mass flow rate increases to the maximum value.

Figure 16 depicts the unsteady Mach number contours at the mid-chord for the same 8 time-steps. Again, limited flow unsteadiness is observed in the vicinity of the damaged blades; however, significant unsteadiness is apparent in the undamaged sector between Blade 15 and Blade 20. In this sector of the fan, the extent of the stall cell progresses from Blade 15 to Blade 20 associated with a decrease in the mass flow rate. Subsequently, the stall cell regresses back to Blade 15 as the mass flow recovers and the flow reattaches. In addition, as the stall cell propagates at the blade tips, the Mach number in the inner span of the corresponding blade passage also decreases, indicating a loss of mass flow through a majority of the blade passage.

The cyclic progression and regression of the stall cell emanating from the damaged sector is similar to the rotating stall phenomenon experienced by undamaged fans and compressors. In a rotating stall event, an upstream flow disturbance causes the suction-side boundary layer of a blade to separate, partially or totally blocking the downstream passage. The flow blockage causes the incoming flow to be diverted to the adjacent blade passages resulting in a decreased angle of attacked for the currently stalled blade and an increased angle of attack for the downstream blade. The decreased angle of attack causes the flow to reattach to the separated blade, and the increased angle of attack causes the downstream blade to stall. The resulting stall cell propagates opposite the direction of rotation at $1 / 3$ to $1 / 2$ the engine rotation speed and typically encompasses only 2 or 3 blades.

In the case of a damaged fan, the separated flow originating from the damaged sector initiates a progressing stall cell similar to a rotating stall cell; however, the substantial leading edge deformation of the damaged blades prevent the flow from reattaching to the upstream blades. As a result, the flow is blocked at an increasing number of blade passages as the stall cell propagates, causing a decrease in the overall mass flow rate. As the mass flow rate decreases, the angle of attack of the blades is decreased such that the flow reattaches and the mass flow rate recovers. The cyclic propogation of the stall cell occurs at roughly $1 / 6$ the engine rotation speed.

\section{Concluding Remarks}

In this study, a CFD-based aerodynamic model suitable for use in an aeroelastic response calculation of a bird-damaged turbofan is presented. The aerodynamic model takes advantage of the commercially available ANSYS CFX code, which is used to perform the steady-state and unsteady CFD calculations for an undamaged and bird-damaged fan. Three computational mesh densities (coarse, medium, and fine) are considered to assess the effects of boundary layer and tip gap resolution on the steady-state aerodynamic calculations. The aerodynamic calculation performed with ANSYS CFX for an undamaged fan stage is verified against results obtained with an industry CFD solver. The increased boundary layer and tip gap resolution of the medium and fine meshes show negligible effect on the steady-state solutions of an undamaged fan. 
The aerodynamic model is used to investigate the steady-state and unsteady aerodynamic behavior of a bird-damaged fan stage. The damage resembles experimental bird strike damage and covers a sector of 5 fan blades in which the damage includes gross leading-edge deformation and global bending and untwist of the blades. An automated mesh deformation procedure is developed that utilizes RBF interpolation to deform an undamaged computational mesh to coincide with the damaged geometry. The mesh deformation procedure can accommodate a variety of damaged configurations, and the resulting deformed mesh retains a high level of mesh quality.

The predicted steady-state flow loss of $14.7 \%$ agrees well with that calculated by the industry solver. In addition, the increased boundary layer and tip gap resolution has limited effect on the predicted flow loss. The characteristic curve indicates that the damaged fan is operating near stall where unsteadiness in the flow is significant. The steady-state calculations show substantial separation and recirculation of the flow at the damaged blades as well as stalled flow at the blade tips for a majority of the fan wheel.

The unsteady calculations of the damaged fan exhibit a periodic solution in which the mass flow rate and total pressure ratio oscillate at a rate of $1 / 6$ the engine rotation speed. Furthermore, the mean flow loss of $17.0 \%$ is slightly greater than that from the steady state solution, indicating that unsteady effects further contribute to the flow losses of the bird-damaged engine. The unsteady flow field exhibits considerable unsteadiness, particularly in the region opposite the damaged sector where a stall cell emanating from the damaged sector progresses and regresses at the blade tips once per oscillation of the unsteady solution.

In a follow up study, the aerodynamic model will be coupled with a finite element based structural solver to determine the effect of bird damage on the forced response and aeroelastic behavior of a bird damaged fan.

\section{References}

${ }^{1}$ Teichmann, H. C. and Tadros, R. N., "Analytical and Experimental Simulation of Fan Blade Behavior and Damage Under Bird Impact," Transactions of the ASME, Vol. 113, October 1991, pp. 582-594.

${ }^{2}$ Isomura, K. and Giles, M. B., "A Numerical Study of Flutter in Transonic Fan," Transactions of the ASME, Vol. 120, July 1998 , pp. 500-507.

${ }^{3}$ Horsley, J., "The 'Rolls-Royce' Way of Validating Fan Integrity," AIAA/SAE/ASME/ASEE 29th Joint Propulsion Conference and Exhibit, Monterey, CA, June 1993, pp. 1-22, Netherlands Association of Aeronautical Engineers.

${ }^{4}$ Kim, M., Vahdati, M., and Imregun, M., "Aeroelastic Stability Analysis of a Bird-Damaged Aeroengine Assembly," Journal of Aerospace Sciences and Technologies, Vol. 5, No. 7, 2001, pp. 469-482.

${ }^{5}$ Bendiksen, O. O., "Aeroelastic Problems in Turbomachines," Transactions of the ASME, Vol. 113, October 1991, pp. 582594.

${ }^{6}$ Bendkisen, O. O., Kielb, R. E., and Hall, K. C., "Turbomachinery Aeroelasticity," Encyclopedia of Aerospace Engineering, John Wiley \& Sons, 2010.

${ }^{7}$ Eschenfelder, P., "Jet Engine Certification Standards," Proceedings of the International Bird Strike Committee, Amsterdam, April 2000, pp. 535-540.

${ }^{8}$ Howard, W. D., "Turbofan Engine Bird Ingestion Testing," Proceedings of the 27th Joint Propulsion Conference, Sacramento, CA, June 1991, AIAA Paper No. 1991-2380.

${ }^{9}$ Imregun, M. and Vahdati, M., "Aeroelastic Analysis of a Bird-Damaged Fan Assembly Using a Large Numerical Model," The Aeronautical Journal, December 1999, pp. 569-578.

${ }^{10}$ Goyal, V. K., Huertas, C. A., Borrero, J. R., and Leutwiler, T. R., "Robust Bird-Strike Modeling Based on ALE Formulation Using LS-DYNA," Proceedings of the 47th AIAA/ASME/ASCE/AHS/ASC Structures, Structural Dynamics, and Materials Conference, Newport, RI, May 2006, AIAA Paper No. 2006-1759.

${ }^{11}$ Hirschbein, M. S., "Bird Impact Analysis Package for Tubine Engine Fan Blades," Proceedings of the 23rd AIAA/ASME/ASCE/AHS/ASC Structures, Structural Dynamics, and Materials Conference, New Orleans, LA, May 1982, AIAA Paper No. 1982-696.

${ }^{12}$ Mao, R., Meguid, S. A., and Ng, T. Y., "Finite Element Modeling of a Bird Striking an Engine Fan Blade," Journal of Aircraft, Vol. 44, No. 2, 2007, pp. 583-596.

${ }^{13}$ Mao, R., Meguid, S. A., and Ng, T. Y., "Transient Three Dimensional Fintie Element Analysis of a Bird Striking a Fan Blade," International Journal of Mechanics and Materials in Design, Vol. 4, No. 1, 2008, pp. 79-96.

${ }^{14}$ Meguid, S. A., Mao, R., and Ng, T. Y., "FE Analysis of Geometry Effects of an Artificial Bird STriking an Aeroengine Fan Blade," International Journal of Impact Engineering, Vol. 35, No. 6, 2008, pp. 487-498.

${ }^{15}$ Nizampatnam, L. S. and Horn, W. J., "Investigation of Equation of State Models for Predicting Bird Impact Loads," Proceedings of the 44th AIAA Aerospace Sciences Meeting and Exhibit, Reno, NV, January 2008, AIAA Paper No. $2008-682$.

${ }^{16}$ Shimamura, K., Shibue, T., and Grosch, D. J., "Numerical Simulation of Bird Strike Damaged on Jet Engine Fan Blade," Proceedings of the ASME/JSME Pressure Vessels and Piping Conference, San Diego, CA, July 2004.

${ }^{17}$ Aotsuka, M. and Tsuchiya, N., "Numerical Simulation of Transonic Fan Flutter with 3-D N-S CFD Code," Proceedings of the ASME Turbo Expo 2008: Power for Land, Sea, and Air, Berlin, Germany, June 2008.

${ }^{18}$ Marshall, J. G. and Imregun, M., "A Review of Aeroelasticity Methods with Emphasis on Turbomachinery Applications," Journal of Fluids and Structures, Vol. 10, 1996, pp. 237-267.

18 of 21 
${ }^{19}$ Bohari, B. and Sayma, A., "CFD Analysis of Effects of Damage Due to Bird Strike on Fan Performance," Proceedings of the ASME Turbo Expo 2010: Power for Land, Sea, and Air, Glasgow, UK, June 2010.

${ }^{20}$ Morris, A., Allen, C., and Rendall, T., "CFD-based Optimization of Aerofoils Using Radial Basis Functions for Domain Element Parameterization and Mesh Deformation," International Journal for Numerical Methods in Fluids, Vol. 58, No. 8, 2008, pp. 827-860.

${ }^{21}$ van Zuijlen, A., de Boer, A., and Bijl, H., "Higher-order Time Integration Through Smooth Mesh Deformation for 3D Fluid-Structure Interaction Simulations," Journal of Computational Physics, Vol. 224, No. 1, 2007, pp. 414-430.

${ }^{22}$ Jakobsson, S. and Amoignon, O., "Mesh Deformation Using Radial Basis Functions for Gradient Based Aerodynamic Shape Optimization," Computers and Fluids, Vol. 36, No. 6, 2007, pp. 1119-1136.

${ }^{23}$ Buhmann, M. D., Radial Basis Functions: Theory and Implementations, Cambridge University Press, Cambridge, 2003.

${ }^{24}$ de Boer, A., van der Schoot, M., and Bijl, H., "Mesh deformation based on radial basis function interpolation," Computers 86 Structures, Vol. 85, 2007, pp. 784-795.

${ }^{25}$ Lamorte, N. and Friedmann, P. P., "Hypersonic Aeroelastic Stability Boundary Computations using Radial Basis Functions for Mesh Deformation," Proceedings of the 18th AIAA/3AF International Space Planes and Hypersonic Systems and Technologies Conference, Tours, France, September 2012.

${ }^{26}$ Carstens, V., Kemme, R., and Schmitt, S., "Coupled Simulation of Flow-Structure Interaction in Turbomachinery," Aerospace Science and Technology, Vol. 7, 2003, pp. 298-306.

${ }^{27}$ Jennions, I. K. and Turner, M. G., "Three-Dimensional Navier-Stokes Computations of Transonic Fan Flow Using an Explicit Flow SOlver and an Implicit k- $\epsilon$ Solver," ASME Journal of Turbomachinery, Vol. 116, April 1993, pp. 261-272. 


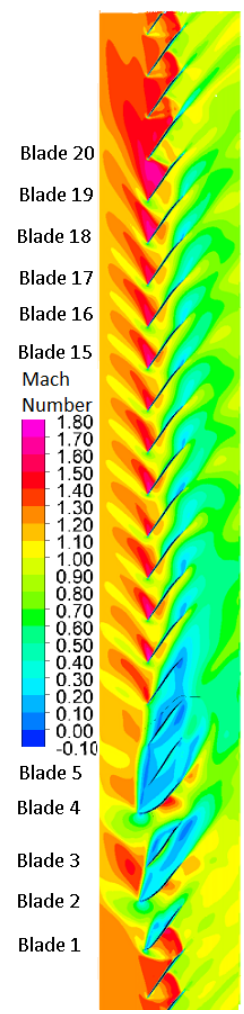

(a) 20.0

revolutions.

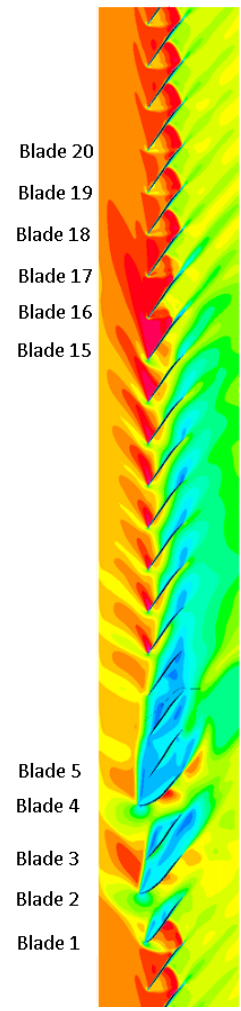

(e) 23.6

revolutions.

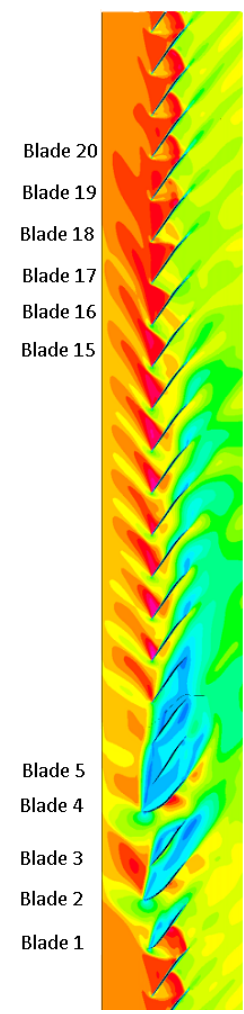

(b) 20.9

revolutions.

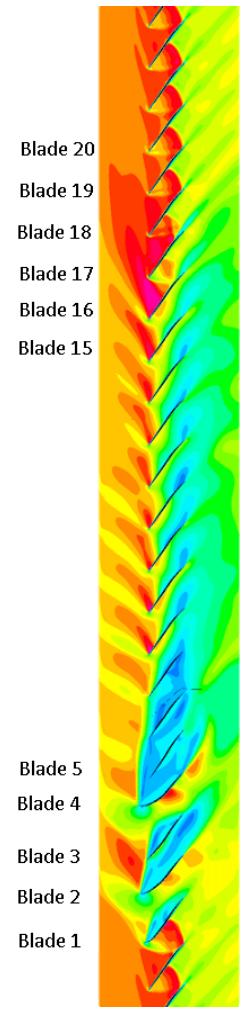

(f) 24.5revolutions.

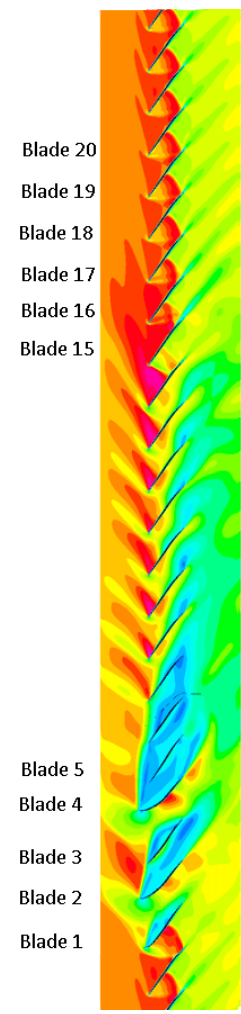

(c) 21.8

revolutions.

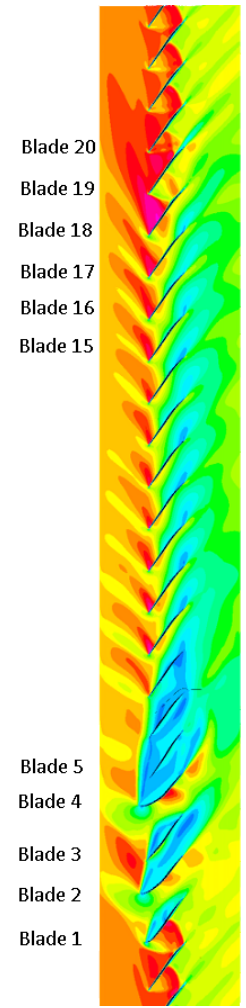

(g) 25.4

revolutions.

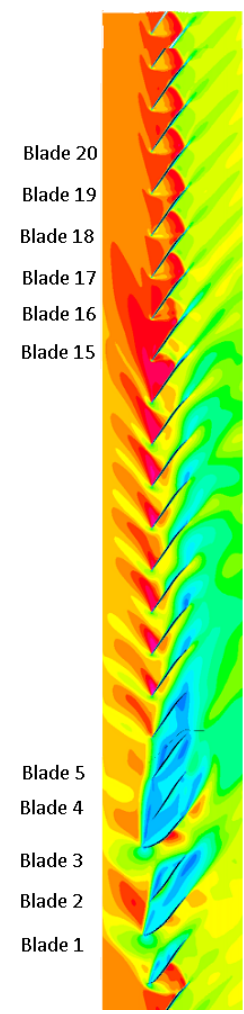

(d) 22.7

revolutions.

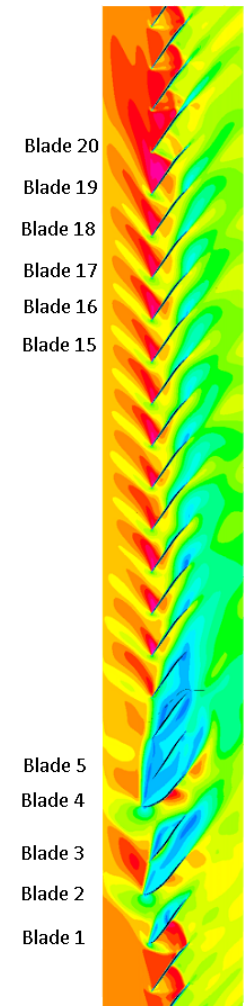

(h) 26.3

revolutions.

Figure 15: Unsteady Mach number contours of the damaged fan at 77\% span over one period of oscillation. 


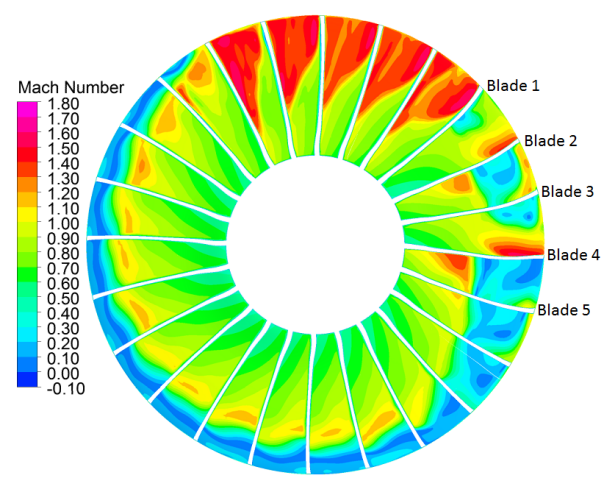

(a) 20.0 revolutions.

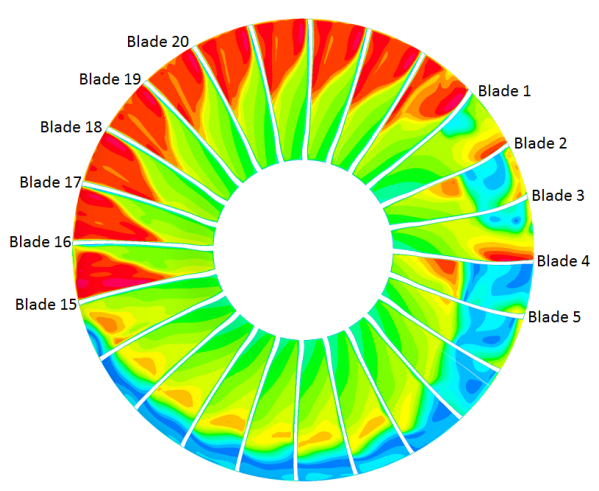

(c) 21.8 revolutions.

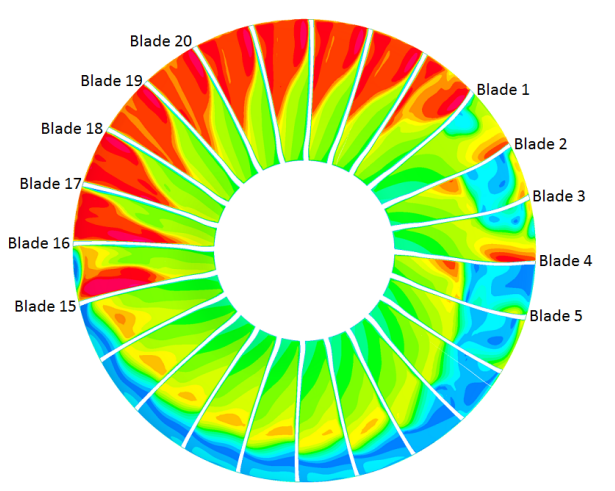

(e) 23.6 revolutions.

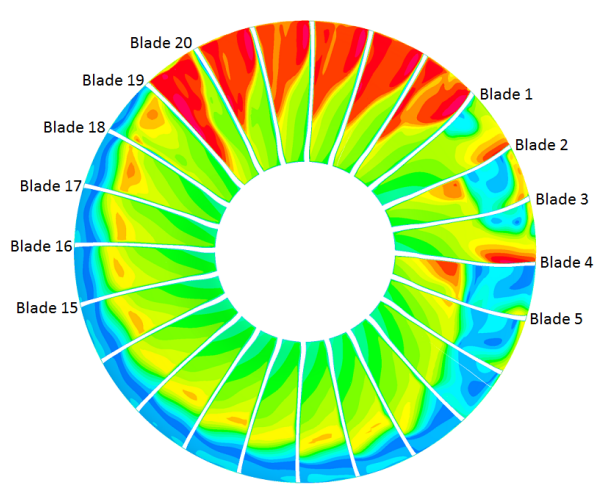

(g) 25.4 revolutions.

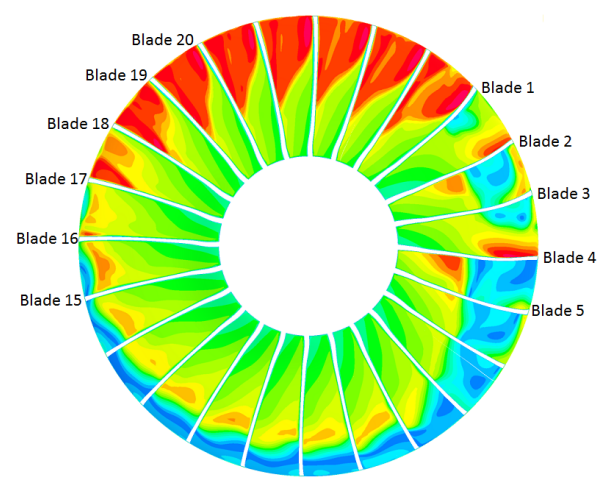

(b) 20.9 revolutions.

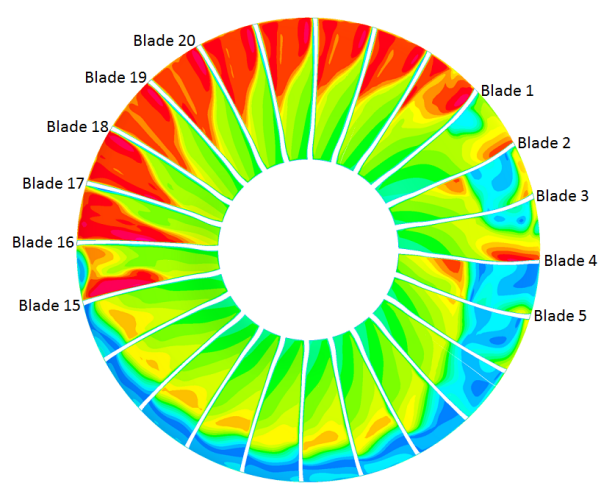

(d) 22.7 revolutions.

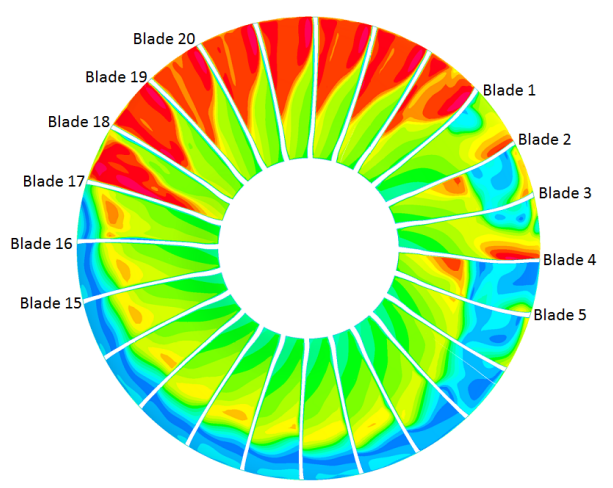

(f) 24.5 revolutions.

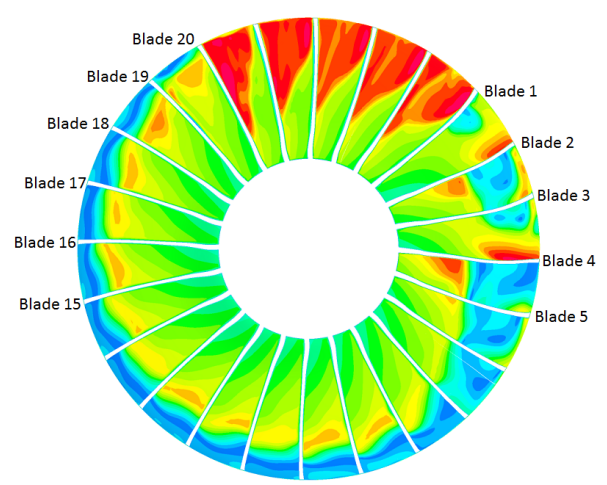

(h) 26.3 revolutions.

Figure 16: Unsteady Mach number contours of the damaged blade at mid-chord over one period oscillation (direction of rotation: counter-clockwise). 